\title{
Crystal structure of FabZ-ACP complex reveals a dynamic seesaw-like catalytic mechanism of dehydratase in fatty acid biosynthesis
}

\author{
Lin Zhang ${ }^{1,2}$, Jianfeng Xiao ${ }^{3}$, Jianrong $\mathrm{Xu}^{1}$, Tianran $\mathrm{Fu}^{1}$, Zhiwei $\mathrm{Cao}^{1}$, Liang Zhu ${ }^{1,2}$, Hong-Zhuan Chen ${ }^{1,2}$, \\ Xu Shen ${ }^{3}$, Hualiang Jiang ${ }^{3}$, Liang Zhang ${ }^{1,2}$ \\ ${ }^{I}$ Department of Pharmacology and Chemical Biology, Shanghai Jiao Tong University School of Medicine, Shanghai, China; \\ ${ }^{2}$ Shanghai Universities Collaborative Innovation Center for Translational Medicine, Shanghai, China; ${ }^{3}$ State Key Laboratory of \\ Drug Research, Shanghai Institute of Materia Medica, Chinese Academy of Sciences, Shanghai 201203, China
}

Fatty acid biosynthesis (FAS) is a vital process in cells. Fatty acids are essential for cell assembly and cellular metabolism. Abnormal FAS directly correlates with cell growth delay and human diseases, such as metabolic syndromes and various cancers. The FAS system utilizes an acyl carrier protein (ACP) as a transporter to stabilize and shuttle the growing fatty acid chain throughout enzymatic modules for stepwise catalysis. Studying the interactions between enzymatic modules and ACP is, therefore, critical for understanding the biological function of the FAS system. However, the information remains unclear due to the high flexibility of $\mathrm{ACP}$ and its weak interaction with enzymatic modules. We present here a $2.55 \AA$ crystal structure of type II FAS dehydratase FabZ in complex with holo-ACP, which exhibits a highly symmetrical FabZ hexamer- $\mathrm{ACP}_{3}$ stoichiometry with each $\mathrm{ACP}$ binding to a FabZ dimer subunit. Further structural analysis, together with biophysical and computational results, reveals a novel dynamic seesaw-like ACP binding and catalysis mechanism for the dehydratase module in the FAS system, which is regulated by a critical gatekeeper residue (Tyr100 in FabZ) that manipulates the movements of the $\beta$-sheet layer. These findings improve the general understanding of the dehydration process in the FAS system and will potentially facilitate drug and therapeutic design for diseases associated with abnormalities in FAS.

Keywords: fatty acid biosynthesis; dehydratase; ACP; complex structure

Cell Research (2016) 26:1330-1344. doi:10.1038/cr.2016.136; published online 22 November 2016

\section{Introduction}

Fatty acid biosynthesis (FAS) is one of the most important processes in cells and organisms on earth. Fatty acids are essential for the cellular membrane assembly, energy storage, cell signal transduction and regulation of gene expression [1-5]. Deficiency in the FAS system causes delays in bacterial development and triggers programmed cell death in plants $[2,3,6]$. In human, deregulated FAS system has been correlated with metabolic syndrome (MetS) diseases, including obesity and typeII diabetes [7-11]. Moreover, human fatty acid synthase

*Correspondence: Liang Zhang

E-mail: liangzhang2014@sjtu.edu.cn

Received 3 June 2016; revised 18 August 2016; accepted 7 September 2016; published online 22 November 2016
(hFASN) has been considered as an oncogene because it is significantly overexpressed in several human cancers with poor prognosis and survival, particularly, including prostate and breast cancers [12-17].

The chemical logic of FAS system is highly conserved between eukaryotes and bacteria except for the arrangement of functional enzymatic modules. It utilizes a series of enzymatic modules to iteratively carry out fatty acid elongation, which is divided into two stages: synthesis initiation and elongation (Figure 1). The initiation stage covalently attaches a short acetyl (or malonyl) group to the terminal sulfhydryl of the 4-phosphopantetheine arm (4'-Pan-arm, hereafter) on the substrate carrier protein, which is a holo-acyl carrier protein (holo-ACP). Subsequently, ACP shuttles among approximately four enzymatic modules in the elongation cycle to process fatty acid extension; and two additional carbons are added 


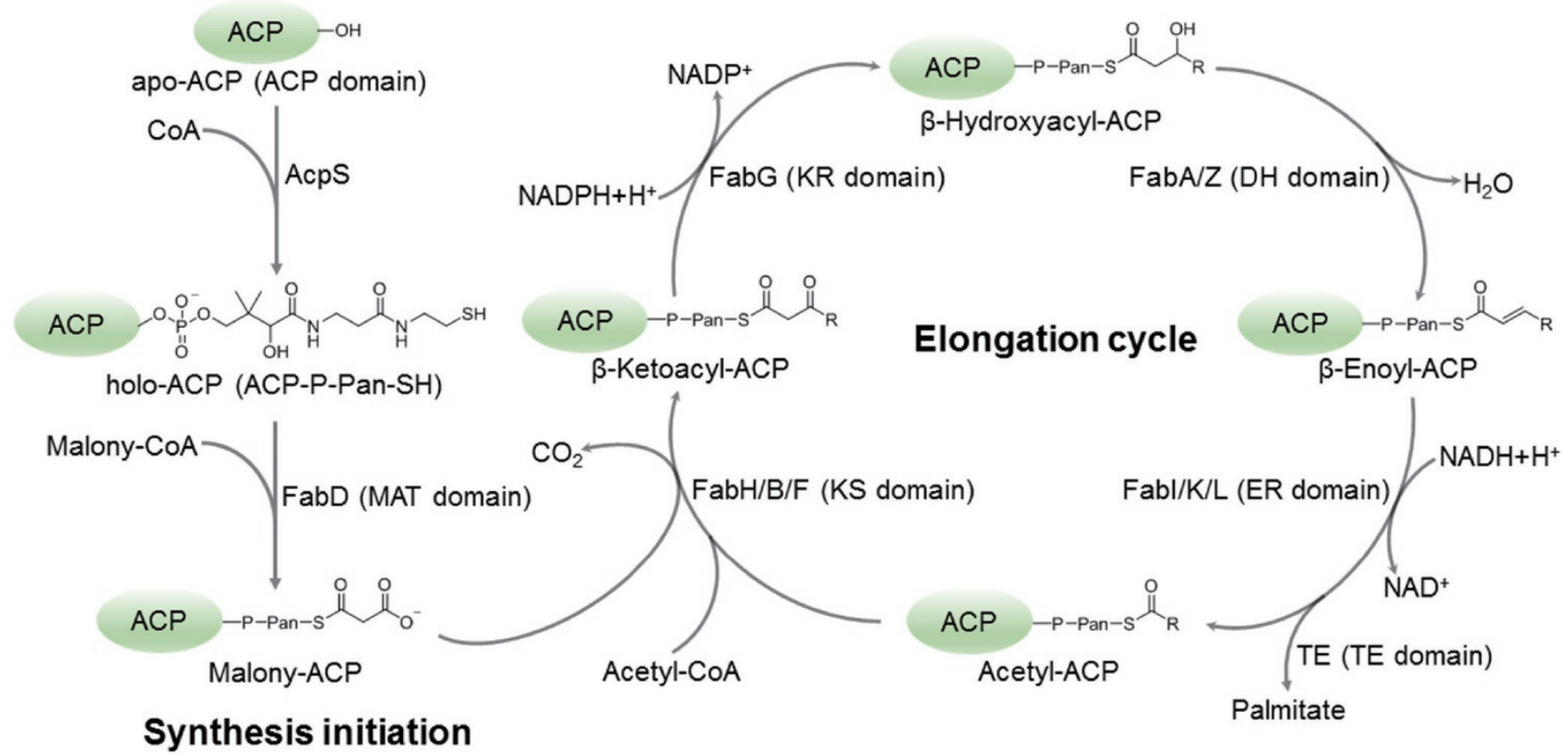

Figure 1 Schematic diagram of fatty acid biosynthesis process. The catalytic process is divided into initiation and elongation stages. In the initiation stage, apo-form of acyl carrier protein (apo-ACP, green) is covalently installed with a 4'-phosphopantetheine moiety (4'-Pan-arm) by holo-ACP synthase (AcpS), forming holo-ACP. Subsequently, a short acetyl (or malonyl) group is covalently attached to the terminal sulfhydryl of the 4'-Pan-arm by malonyl-CoA-/acetyl-CoA-ACP transacylase (MAT domain in FAS-I; FabD in FAS-II). The elongation cycle mainly includes four catalytic steps to process fatty acid extension successively. The first step is decarboxylative condensation to form $\beta$-ketoacyl-ACP by $\beta$-ketoacyl synthase (KS domain in FAS-I; FabH, FanB and FabF in FAS-II); the second step is NADPH-dependent reduction of $\beta$-ketoacyl-ACP to $\beta$-hydroxyacyl-ACP by $\beta$-ketoacyl reductase (KR domain in FAS-I; FabG in FAS-II); the third step is $\beta$-hydroxyacyl-ACP dehydration to $\beta$-enoyl-ACP by $\beta$-hydroxyacyl-ACP dehydratase (DH domain in FAS-I; FabA and FabZ in FAS-II); the last step is $\beta$-enoylACP reduction by NADPH-dependent $\beta$-enoyl reductase (ER domain in FAS-I; Fabl, FabK and FabL in FAS-II). After four steps, two carbons are added to the fatty acid chain, and the fatty acid chain either re-enter the elongation cycle for further extension or is released from ACP by thioesterase (TE domain in FAS-I; TE in FAS-II) once the length reaches 16-18 carbons.

to the substrate chain in each cycle until the product is released. On the basis of the enzymatic module arrangement, the FAS system is divided into associative and dissociative architectural types. The associative architecture (FAS-I) exists in some bacteria (e.g., CMN-FAS) and eukaryotes, including mammals and fungi $[1,18]$. All the enzymatic modules function as domains on a large polypeptide (one or two polypeptide(s) in fungi), which is encoded by a single gene (one or two gene(s) in fungi). The dissociative architecture (FAS-II) exists in plants, mitochondria and bacteria, whereas the enzymatic modules function as a series of individual soluble enzymes encoded by discrete genes [2]. So far, crystal structures of FAS-I synthases (FASN) from Sus scrofa and fungi [1923] and FAS-II individual enzymes from various bacterial species have been reported [2, 24-28], which provided detailed insights into the biochemical mechanism at an atomic level. However, due to the high flexibility and diffusible characteristics of ACP, the interactions between ACP and enzyme modules in FASN or individual enzymes in FAS-II have rarely been investigated except for an incomplete enoyl reductase (FabI)-ACP structure and a covalently crosslinked $\beta$-hydroxyacyl-ACP dehydratase (FabA)-ACP structure [25, 29]. Hence, the key mechanisms underlying the recognition and processing of ACP by enzymatic modules for substrate catalysis remain unclear, especially in the elongation cycle.

The elongation cycle predominantly consists of four catalytic steps (Figure 1). Dehydration of $\beta$-hydroxyacyl-ACP is the third step, which is processed either by the dehydratase (DH) domain of FAS-I synthase or two individual DH homologs, FabA and FabZ, in FAS-II (Figure 1) [1, 2]. FabA appears as a homodimer and catalyzes the dehydration of short fatty acids ( $\leq 10$-carbon) as well as the isomerization of trans-2-acyl-ACP to cis2-acyl-ACP. In contrast, FabZ appears as a hexamer in 
most cases (dimer is also observed besides hexamer in Plasmodium falciparum) and preferentially catalyzes the dehydration of long-chain fatty acids [2] (Supplementary information, Figure S1). In addition, FabZ is involved in the balance maintenance of fatty acid and lipid A biosynthesis by regulating deacetylase LpxC [30]. Due to their indispensable functions and ubiquitous distributions, FabA and FabZ are regarded as important drug targets against pathogenic microbes. The enzymatic characteristics of FabA and FabZ, as well as their structures in various bacterial species have been well studied, and several lead compounds have been developed [31-35]. In 2014, Nguyen et al. [29] reported the first FabA-ACP complex structure, in which a mechanism-based synthetic alkyne probe was employed to covalently crosslink two ACP molecules to the FabA homodimer. The complex structure reveals ACP binding and the substrate accommodation mechanism of FabA for the first time. A putative ACP binding mechanism was proposed based on the significant conformational changes of ACP helices $\alpha 2$ and $\alpha 3$ observed during binding to FabA. Nevertheless, this structural model has not provided adequate insights into how the DH module recognizes and regulates ACP molecules for enzymatic catalysis in the FAS system and what determines the distinct substrate length preference and isomerization mechanism between FabA and FabZ. The DH module (FabZ)-ACP complex structure in the FAS system presented in current study illustrates a dynamic seesaw-like ACP binding and regulation mechanism of FabZ, representing a novel substrate selection and catalysis mechanism for the DH module in the FAS system.

\section{Results}

\section{Overall structure of the FabZ-ACP complex}

The recombinant FabZ and ACP from Helicobacter pylori were expressed and purified as described previously $[36,37]$. Instead of using an alkyne probe attached to ACP (which was used in FabA-ACP structure [29]), holo-ACP was directly employed in crystallization to obtain the natural conformation of the DH module bound with ACP. The purified FabZ and holo-ACP proteins were mixed with a molar ratio of 1:7, and further purified by using Superdex 200 gel-filtration column. The crystals appeared within 30 days under $277 \mathrm{~K}$ in hanging drop, and the complex structure was determined by molecular replacement (MR) at a resolution of $2.55 \AA$ (Supplementary information, Table S1). The asymmetric crystallographic unit contains a FabZ dimer subunit bound with a holo-ACP molecule. However, the gel-filtration results indicated that the complex appears to have a stable hexamer conformation that reflects native FabZ in complex with ACP (Supplementary information, Figure S2). As shown in Figure 2A, the overall structure of the FabZ-ACP hexamer generated by crystallographic symmetry operation displays a turbine-like shape with six FabZ monomers arranged in a ring-like contact topology to form a classic FabZ "trimer of dimers" hexameric turbine core [26]. Each FabZ dimer subunit is bound by one holo-ACP, resulting in a stoichiometry of (FabZ dimer-ACP) $)_{3}$.

In the dimer subunit, an unexpected 2:1 (FabZ monomer:ACP) stoichiometric binding ratio is observed, where only one ACP molecule binds to a FabZ dimer. FabZ monomers A and B within the dimer subunit adopt a typical $\beta+\alpha$ "hot dog" fold in which a layer consisting of six anti-parallel $\beta$-sheets ( $\beta 1-\beta 6$ strands, hereafter) wraps around the long, central six-turn $\alpha$-helix ( $\alpha 3)$ (Figure $2 \mathrm{~B})[2,26]$. The $\beta$-sheet layer from each monomer further interacts with each other through their $\beta 3$ strands to form a stable FabZ homodimer subunit. Next to the dimer interface, there are two L-shaped substrate-binding and catalysis tunnels, which are $\sim 20 \AA$ away from each other. Each tunnel consists of residues from both monomers. The entrance of the tunnel is located on the $\beta 3$ strand near the dimer interface, and protected by a gatekeeper residue Tyr100 from bulk solvent. Here we named the two tunnels of the dimer subunit tunnels A (the gatekeeper residue Tyr100 is from monomer A) and B (Tyr100 is from monomer B). The holo-ACP binds to the $\beta$-sheet layer right on the FabZ dimer interface through its conserved $\alpha 2$ helix, occupying the monomer $\mathrm{A}$ and $\mathrm{B}$ surface areas of $148.82 \AA^{2}$ and $335.03 \AA^{2}$, respectively, and inserts the 4'-Pan-arm into tunnel B (Figure 2B). The FabZ-ACP interactions are established through multiple hydrogen bonds (H-bonds) between a list of conserved residues, including those between positively charged residue Arg $110^{\prime}$ on FabZ $\beta 3$ strand (the prime indicates the residue from FabZ monomer $\mathrm{A}$ in the dimer, otherwise from monomer B) and two negatively charged residues Asp35 and Asp38 on the N-terminus of ACP $\alpha 2$ helix, between Lys129 on the loop between FabZ $\beta 4$ and $\beta 5$ strands and Glu47 on the C-terminus of ACP $\alpha 2$ helix, and between Lys 152 on FabZ $\beta 6$ strand and Glu41 on the C-terminus of ACP $\alpha 2$ helix (Figure 2B, Supplementary information, Figures S3, S4 and Table S2). In addition to H-bonds, the sidechains of Leu37, Val40 and Met44 on ACP $\alpha 2$ helix form hydrophobic contacts with the hydrophobic patch composed of Met102, Thr103, Ile132 and Lys152 from FabZ (Figure 2B). These hydrophobic and electrostatic interactions orient the ACP $\alpha 2$ helix precisely with the entrance of the FabZ tunnel B. Meanwhile, the sidechain of the gatekeeper residue Tyr100 in FabZ monomer B points toward Lys62' to form an open 
A

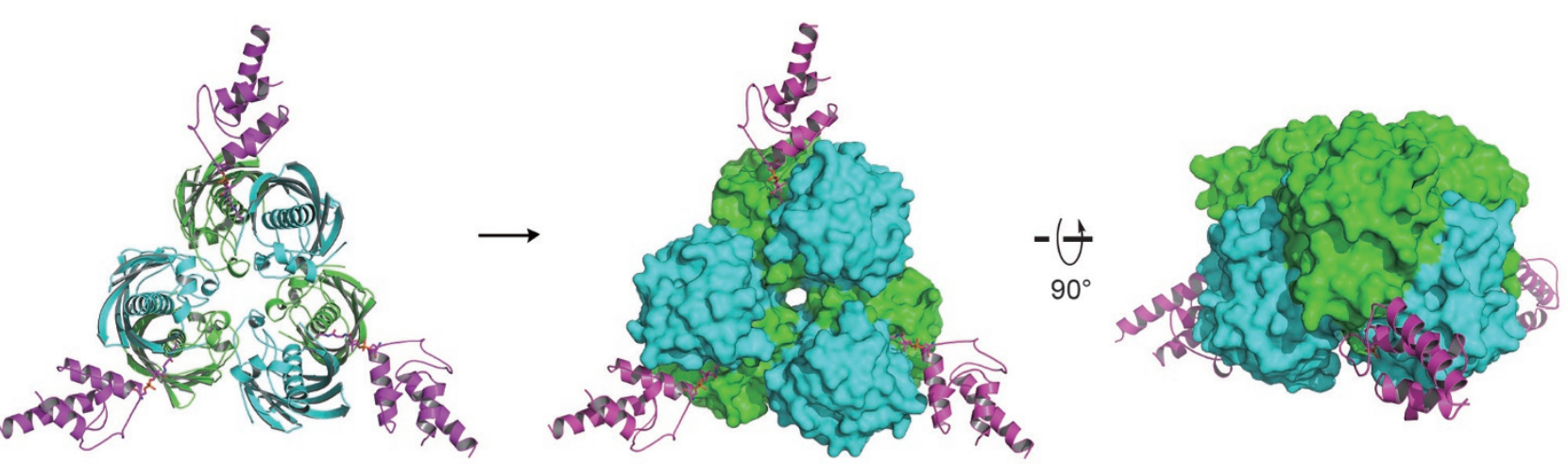

B
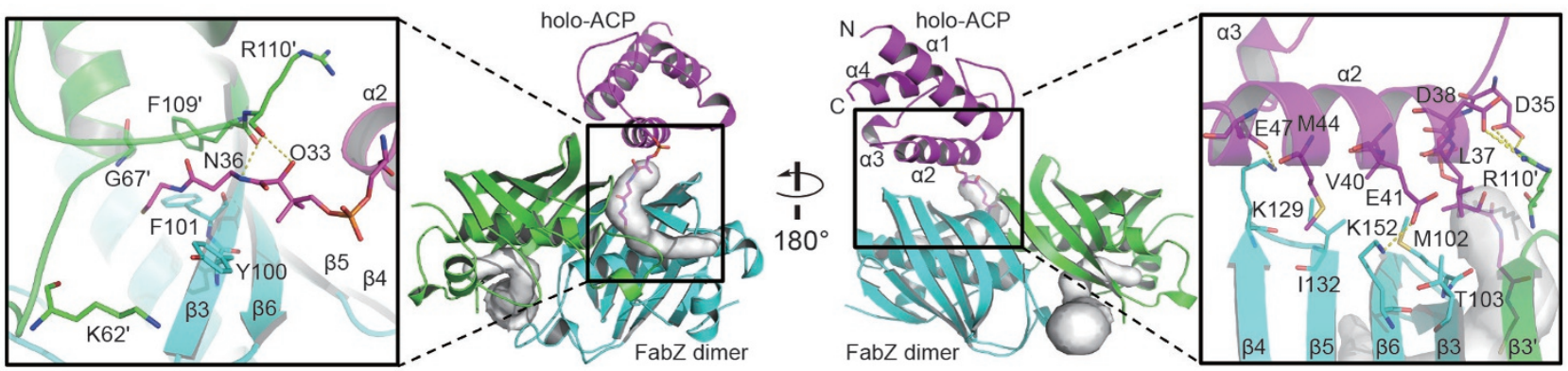

Figure 2 Crystal structure of the FabZ-ACP complex. (A) Overall structure of the FabZ-ACP complex. FabZ dimer subunits are colored in green/cyan, and ACP is colored in magenta. The cartoon was generated by Pymol. (B) Interactions between $\mathrm{ACP}$ and FabZ dimer subunit. White surface of FabZ active tunnel was generated by Pymol plugin Caver. Residues involved in the interactions are shown as sticks, and the prime indicates the residues from FabZ monomer A, otherwise from B. $\mathrm{H}$-bond network is shown as yellow dashes, and secondary structural elements are labeled as well.

conformation so that the ACP 4'-Pan-arm can comfortably enter tunnel B (Figure 2B). Inside tunnel B, the arm displays a nearly extended conformation (Supplementary information, Figure S5). The conformation is stabilized by H-bonds between the backbone oxygen of Arg110' from FabZ and ACP 4'-Pan-arm hydroxyl group (atom $\mathrm{O}^{33}$ ) and the first - $\mathrm{NH}$ group (atom $\mathrm{N}^{36}$ ) (Figure 2B), which indicates that the role of Arg110 in ACP binding is indispensable, consistent with results from previous reports $[2,38]$.

\section{Fatty acyl chain length preference of FabZ}

Previous studies indicate that the L-shaped FabZ active tunnel is not adequate for accommodating a long fatty acid substrate ( $>12$-carbon), although FabZ enzymatically prefers long substrates [2, 26-28]. In the current structure, ACP binding and insertion of the 4'-Pan-arm expand the active tunnel by pushing the $\beta$-sheet layer toward $\beta 4$ (Figure 3A), which evokes a novel Y-shaped tunnel with extended potential for long fatty acid accommodation. The extension of the classical L-shaped tunnel B starts around residue Gly79 by forming two tunnel branches (designated tunnels I and II; Figure 4A). Tunnel I consists of hydrophobic residues Gly78, Gly79, Trp133, Val135, Leu151 and Ala153 (Figure 4B), whereas tunnel II consists of hydrophobic residues Leu21, Phe83, Ile93, Ala94, Lys97 and Ile98 from monomer B (Figure 4C). Accordingly, the hydrophobic nature of both tunnels I and II is suitable for accommodation of fatty acids.

To investigate the selectivity of two tunnels for different lengths of fatty acid substrates, we docked 4-, 6-, 8-, $10-, 12-, 14-$ and 16-carbon fatty acid chains into the active tunnel structure (Supplementary information, Table S3) [39]. As shown in Figure 4, short fatty acid chains (4-, 6-, 8-, 10- and 12-carbon chains) extend to tunnel I, whereas tunnel II is blocked by the sidechain of Phe83 (closed conformation, pointing toward Ile98). In contrast, longer fatty acid chains (14- and 16-carbon chains) extend to tunnel II by switching the sidechain of Phe 83 toward Ile93 to form an open conformation [26]. Notably, the exit of tunnel II is a loop area between $\beta 3$ and $\alpha 4$ (residues Ala94-Lys97), which was reported to be disordered in previously determined FabZ structures [27, 28], suggesting that this exit has flexibility and compatibility 
A

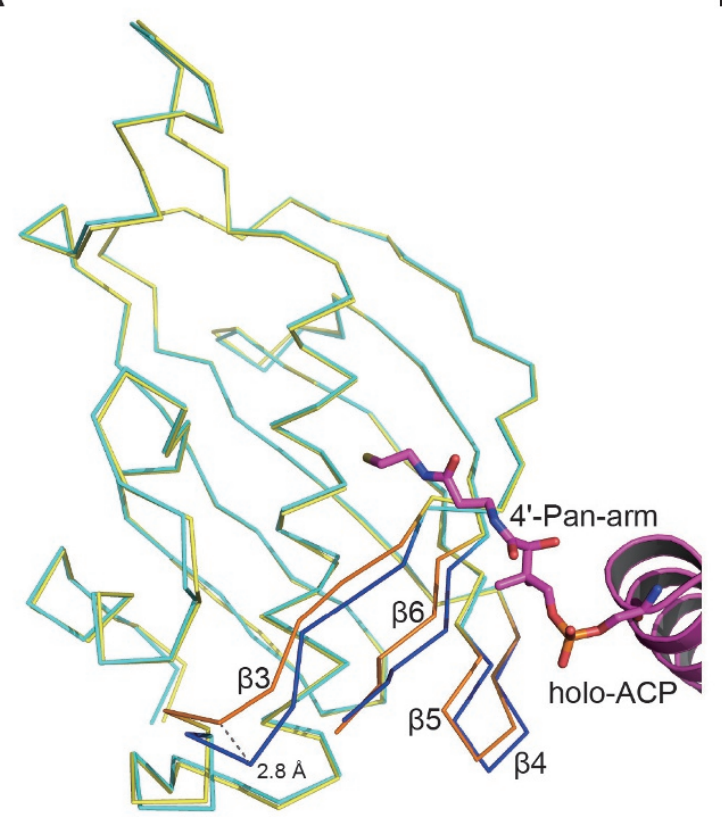

C

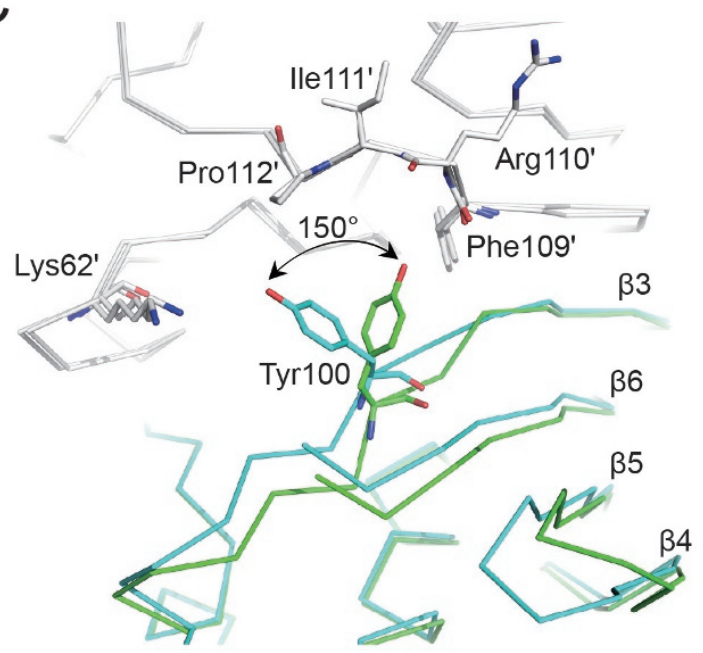

$E$

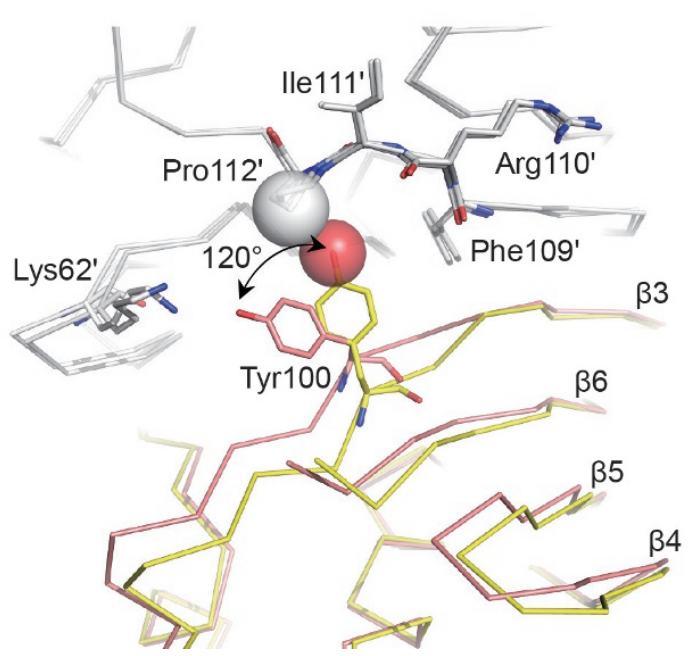

B

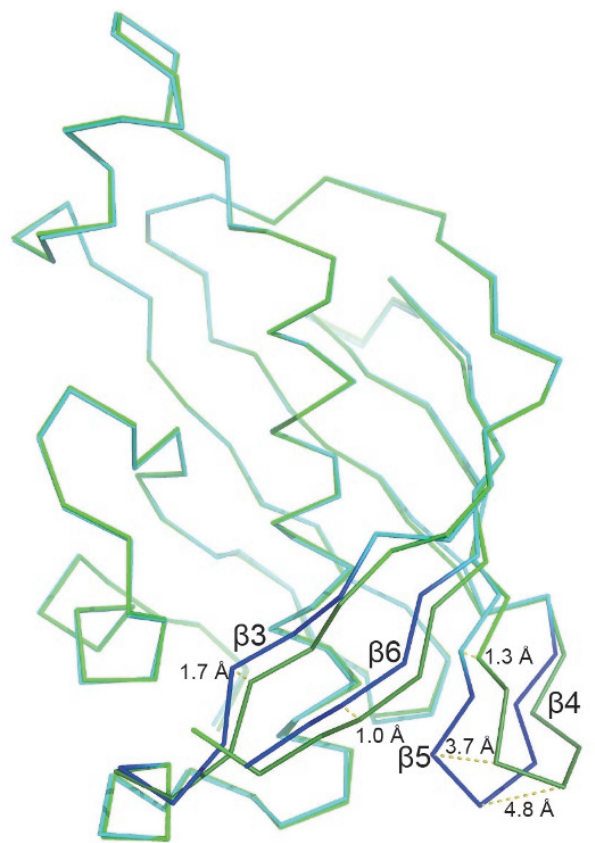

D

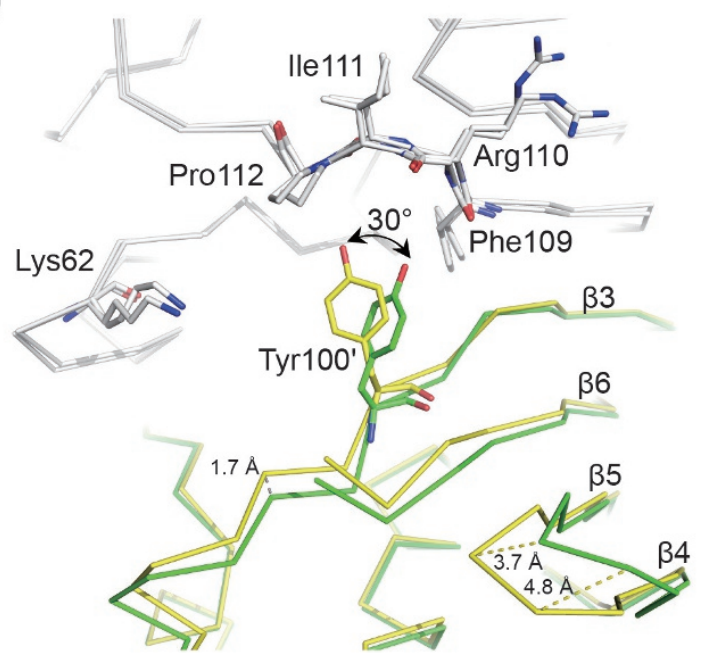

$\mathrm{F}$

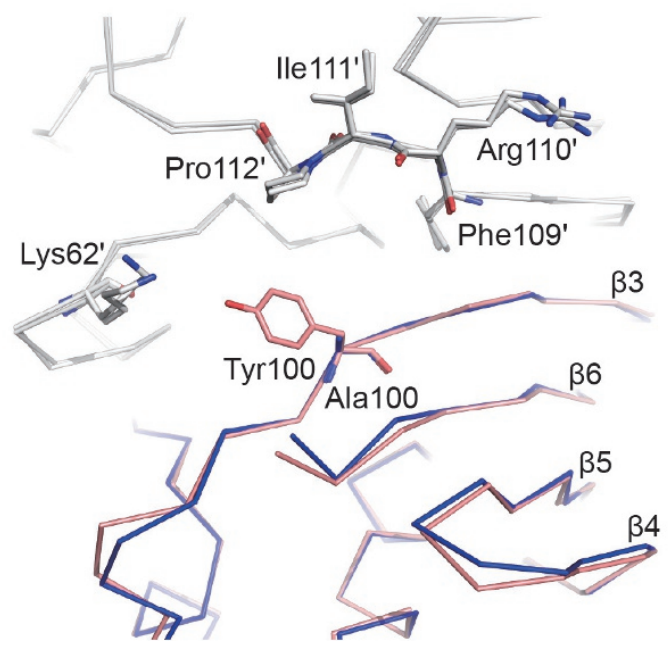

SPRINGER NATURE | Cell Research | Vol 26 No 12 | December 2016 
for threading out long substrates.

\section{Distinct isomerization behavior between FabA and FabZ}

The FabZ dimer shares high structural similarity with the FabA dimer. Nevertheless, unlike FabA, FabZ does not function as an isomerase [2]. Comparison of the structures of FabZ-ACP and FabA-ACP complexes, both docked with an 8-carbon trans-2 fatty acid chain, shows that the ACP 4'-Pan-arms adopt a similar conformation in the two structures, whereas the conformation of the fatty acid substrate appears to be significantly different (Supplementary information, Figure S6). The carbon chain displays a nearly U-shape conformation in FabZ tunnel I compared with the L-shape in FabA because the tunnel leading to a U-shape in FabA is blocked by Val90 and Val162. Interestingly, the fatty acid chain adopts a cis conformation in FabA, whereas it remains the trans configuration in FabZ, which is consistent with a hypothesis proposed by Kimber et al. [27] that the L-shape tunnel has much less steric hindrance for the carbonyl chain isomerization compared with the U-shape.

\section{The dynamic seesaw-like catalytic mechanism of FabZ}

Unlike two ACPs covalently binding to the FabA dimer, only one ACP binds to the FabZ dimer subunit. The superposition of holo-ACP from the FabZ-ACP complex with native apo- and holo-ACP structures displays high structural similarity except for the 4'-Pan-arm orientation (Supplementary information, Figure S7), suggesting that the conformation of ACP itself does not interfere with the stoichiometric binding. In contrast, significant conformational changes in both FabZ monomers were observed. In FabZ monomer B, the anti-parallel $\beta$-sheet layer moves $\sim 2.8 \AA$ toward $\beta 4$ due to ACP binding and 4'-Panarm insertion (Figure $3 \mathrm{~A}$ ). The narrow L-shaped active tunnel is, therefore, expanded to a Y-shape to accommodate a long fatty acid substrate. In addition, the phenol ring of Tyr100 sidechain points toward Lys62' to allow the 4'-Pan-arm inserting into the catalytic tunnel (Figure 3C). Surprisingly, FabZ monomer A also has similar conformational changes upon ACP binding to monomer B. The $\beta$-sheet layer in monomer A moves 1.0-1.7 $\AA$ farther toward $\beta 4$ compared with that in monomer B and the specific loop between $\beta 4$ and $\beta 5$ is extruded by a distance of $\sim 4.8 \AA$ (Figure $3 \mathrm{~B}$ ). The extruded loop introduces clashes into most of interactions between the $\beta$-sheet layer of FabZ and ACP $\alpha 2$ helix, especially the hydrophobic contacts between FabZ Lys129' and Glu47/Glu53 from ACP, as well as FabZ Ile132' and Val40/Met44 from ACP (Supplementary information, Figure S8), forming a conformation unfavorable for ACP binding. To support such conformational movements, the Tyr $100^{\prime}$ sidechain adopts an abnormal closed conformation by further rotating $\sim 30^{\circ}$ clockwise along the $\mathrm{C}_{\alpha}-\mathrm{C}$ bond from the regular closed conformation (in the native FabZ hexamer structure [26]) and pointing toward the backbone carbonyl oxygen of Phe109 to generate additional H-bond (Figure 3D). These structural observations strongly argue that FabZ regulates ACP binding and releasing through alternating movements of the $\beta$-sheet layer.

Indeed, these $\beta$-sheet layer movements have been observed in our previous study of the native FabZ structure, in which three monomers (defined as monomers A-C) adopt a close conformation, while the other three (monomers D-E) adopt an open conformation [26]. These movements directly correlated with the sidechain position of the gatekeeper residue Tyr100. Tyr100 is located on the $\beta 3$ strand next to the active tunnel entrance and adopts two distinct conformations in the native FabZ hexamer structure either by pointing the phenol ring toward Lys62' to form an open conformation (the tunnel entrance is opened to substrate; e.g., monomer E) or rotating the phenol ring along the $\mathrm{C}_{\alpha}-\mathrm{C}_{\beta}$ bond for $\sim 120^{\circ}$ to interact with Pro112' to form a closed conformation (the tunnel entrance is blocked by the sidechain of Tyr100; e.g., monomer A) (Figure 3E). In the native

Figure 3 Superposition of FabZ monomer subunits. In the structure of a FabZ-ACP dimer subunit, only one monomer is associated with ACP, termed monomer B; the other is termed monomer A. In the structure of the ACP-unbound FabZ hexamer (PDB code: $2 \mathrm{GLL}$ ), three monomers adopt open conformation (monomers $\mathrm{D}, \mathrm{E}$ and $\mathrm{F}$ ), and the other three adopt close conformation (monomers A, B and C). (A) Superposition of FabZ monomer B (cyan) from FabZ-ACP complex with monomer E (yellow) from native FabZ hexamer structure (PDB code: $2 G L L$ ). The $\beta$-sheet layers from monomer $B$ and $E$ are colored in blue and orange, respectively. holo-ACP is colored in magenta, and the 4'-Pan-arm is shown in sticks. (B) Superposition of FabZ monomers A (green) and B (cyan) from the FabZ-ACP complex. The $\beta$-sheet layers from monomers A and B are colored in forest green and blue, respectively. (C-F) Superposition of FabZ $\beta$-sheet layers. Residues around the entrance are shown and labeled. (C) Superposition of monomer A (green) with monomer B (cyan) from FabZ-ACP complex. (D) Superposition of monomer A (yellow) from the native FabZ hexamer (PDB code: 2GLL) with monomer A (green) from the FabZ-ACP complex. (E) Superposition of monomer A (yellow) with monomer $E$ (wheat), both from native FabZ hexamer structure. Atoms of Tyr100 and Pro112' involved in the interactions are presented as transparent spheres. (F) Superposition of monomer $E$ (wheat) from the native FabZ hexamer with monomer A (blue) from FabZ (Y100A) mutant hexamer (PDB code: 2GLV). 
A

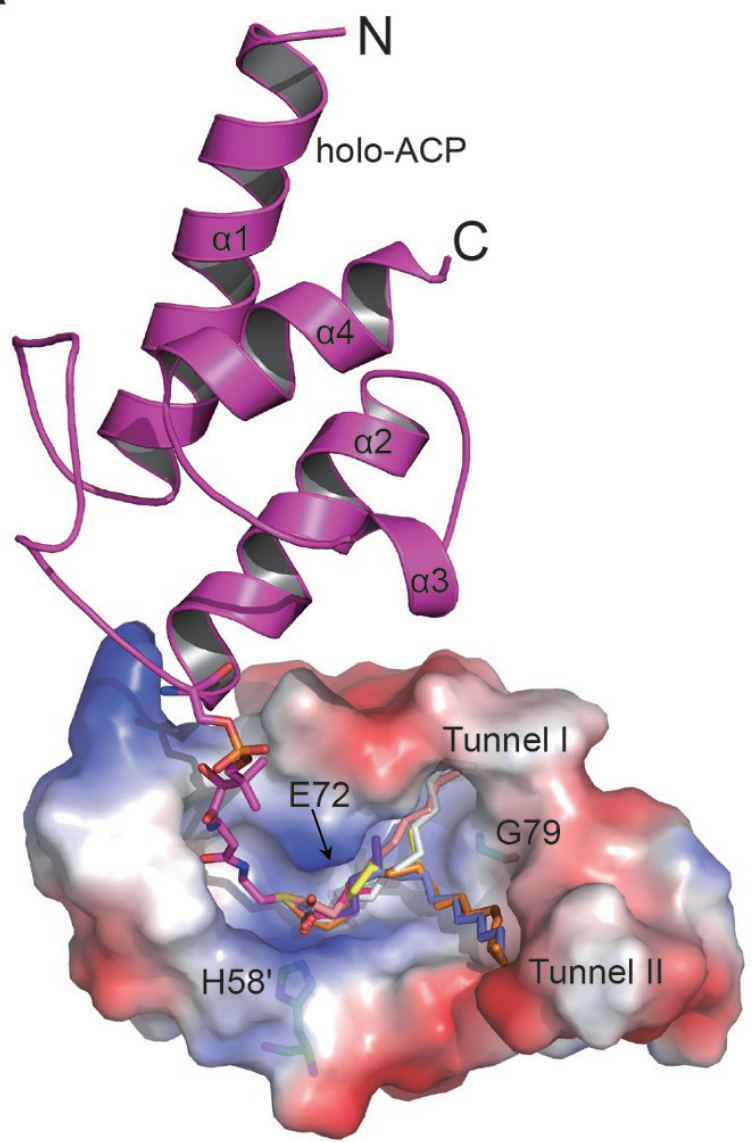

B

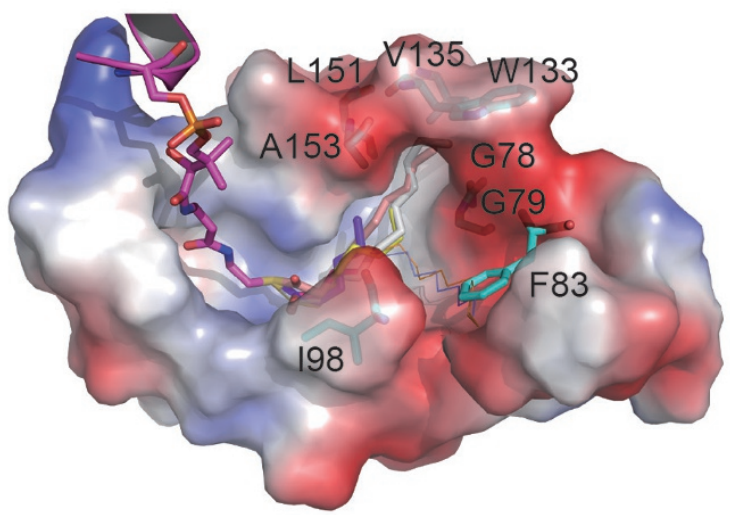

C

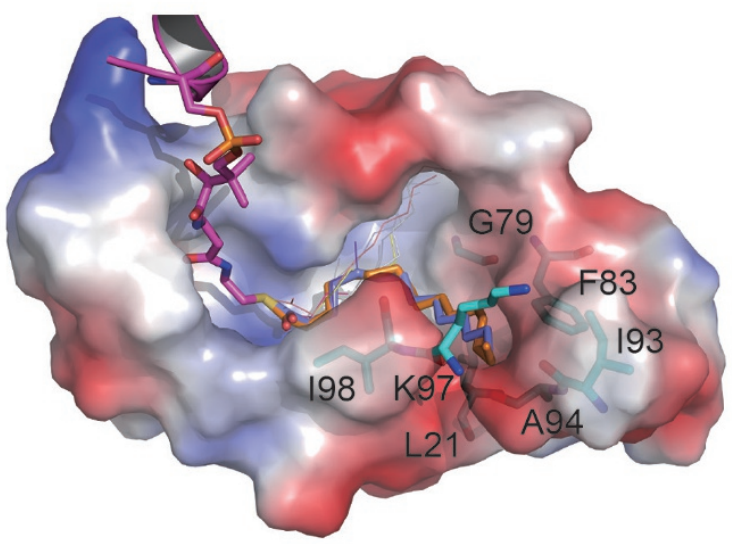

Figure 4 Side view of FabZ active tunnel B surface with fatty acids of different lengths modeled to ACP. The electrostatic surface and ribbons were generated by Pymol. The color range from red (negative) to blue (positive) represents the surface electrostatic potentials of $-75 \mathrm{e} / \mathrm{kT}$ to $+75 \mathrm{e} / \mathrm{kT}$. ACP is colored in magenta. Fatty acid chains with $4,6,8,10,12,14$ and 16 carbons are colored in hot pink, purple blue, yellow, salmon, gray, slate and orange, respectively. Secondary structural elements, tunnels I and II are labeled. (A) Side view of FabZ active tunnel B surface with fatty acids of different lengths modeled to ACP. Catalytic residues His58' and Glu72, and residue Gly79 in the Y-shaped tunnel fork are shown as sticks. (B) Side view of FabZ active tunnel surface with short fatty acid chains (4-12 carbons). Short fatty acid chains and residues involved in the interactions are shown as sticks, and long chains with 14 and 16 carbons are shown as lines. The closed conformation of backdoor residue Phe83 (pointing toward lle98) shown here is replaced by the corresponding residue from native $\mathrm{HpFabZ}$ structure (PDB code: 2GLL). It blocks long fatty chain (shown as a line) stretching to tunnel II. (C) Side view of FabZ active tunnel surface with long fatty acid chains (14 and 16 carbons). Long fatty acid chains and residues involved in the interactions are shown as sticks, and short chains are shown as lines. Phe83 adopts an open conformation (pointing to lle93) and allows long fatty chains (shown as sticks) to stretch into tunnel II.

FabZ hexamer structure, the hydrophobic interaction between the Tyr100 sidechain and Pro112' in the closed conformation pushes the $\beta$-sheet layer further toward $\beta 4$ compared with the open conformation (Figure 3E). In the FabZ-ACP complex structure, the abnormal conformation of Tyr $100^{\prime}$ sidechain further pushes the $\beta$-sheet layer toward $\beta 4$ (Figure 3D), likely causing ACP releasing. Previous studies suggested that the gatekeeper residue commonly exists in the DH across species in the FAS system for protecting the hydrophobic catalytic tunnel from bulk solvent $[2,27,28]$. Multiple sequence alignments of FabZ among 72 bacterial species indicate that tyrosine is the predominant gatekeeper $(61 \%)$, whereas some other residues with hydrophobic sidechains, such as phenylalanine $(22 \%)$ and leucine $(17 \%)$, are also observed (Supplementary information, Figure S3C). Our studies suggest that the absence of the Tyr100 sidechain in FabZ Y100A mutant completely abolishes the move- 
ments of the $\beta$-sheet layer within the FabZ hexamer (Figure $3 \mathrm{~F}$ ) and significantly reduces FabZ enzymatic activity to $\sim 10 \%$ by interfering with the release of ACP [26]. Together with structural observations, these results indicate that the hydrophobic sidechain of such a gatekeeper is indispensable for the maintenance of FabZ enzymatic activity not only for simply protecting the hydrophobic catalytic tunnel from bulk solvent but also for regulating $\mathrm{ACP}$ binding and releasing by rotating its sidechain to manipulate the movements of the $\beta$-sheet layer in an alternating manner like a seesaw.

\section{Biophysical studies of FabZ-ACP binding}

The structural packing analysis indicates that the $\mathrm{N}$-terminus of holo-ACP from the crystallographic neighbor FabZ-ACP molecule has contacts with the ACP-unbound FabZ monomer A to form a potential large tetramer complex with a stoichiometry of (FabZ hexamer- $\left.\mathrm{ACP}_{3}\right)_{4}$ or an even larger aggregated mass due to the linkage effect (Supplementary information, Figure S9). To investigate whether the crystal packing force from the neighbor ACP molecule contributes to the conformational changes in monomer A observed in our crystal structure, we carefully examined the contacts between monomer A and the neighbor ACP. The contacts are contributed by hydrophobic interactions between the sidechains of Met102' and Met154' from FabZ monomer $\mathrm{A}$ and the backbones of a cloning-generated extra residue $\operatorname{Gly}(-2)$ at the $\mathrm{N}$-terminus of $\mathrm{ACP}$, as well as the sidechain of Arg110 from FabZ monomer B and the sidechain of another cloning-generated extra residue $\operatorname{Tyr}(-1)$ of ACP (Supplementary information, Figure S9A and S9B). Among the interactions, Tyr100' from FabZ monomer A interacts weakly through its hydroxyl group to the backbone N atom of ACP Ser(-4) residue ( $\sim 4.0 \AA$ distance). As $\operatorname{Ser}(-4)$ is the very first residue at the N-terminus of ACP, the interaction between Ser(4) and Tyr100 is even weaker than normal hydrophobic interaction due to the high flexibility of the residue. The superposition of FabZ monomers A and B suggests that the sidechains of Met102 and Met154 are slightly bended, and the sidechain of $\operatorname{Arg} 110$ is extended due to the packing forces (Supplementary information, Figure S9C and S9D). Obviously, the packing forces tend to push the sidechain of Tyr100' from monomer A anticlockwise, forming an open conformation rather than the current "super-close" conformation, and push the $\beta$-sheet layer to move backward rather than toward $\beta 4$. Hence, the crystal packing has little influence on the observed conformational changes in monomer $\mathrm{A}$ in the complex structure.

As these interactions, potentially induced by crystallographic packing, occur close to the entrance of FabZ tunnel A, steric hindrance of the neighbor holo-ACP may block the FabZ tunnel entrance, thus preventing the binding of a second holo-ACP in solution (Supplementary information, Figure S2). To eliminate this concern, the oligomeric state of the FabZ-ACP complex in solution was evaluated by size-exclusion chromatography coupled with multi-angle light scattering (SEC-MALS). Compared with native FabZ, the molecular weight of the complex is almost exactly that of a FabZ hexamer plus three holo-ACPs (Figure 5A and 5B). The stoichiometry was further confirmed by synchrotron-based small-angle $\mathrm{X}$-ray scattering (SAXS) (Figure 5C-5E). The low-resolution particle model reconstructed from the SAXS experimental profiles fits well with the respective complex structure $\left(\chi^{2}=0.922\right)$, which suggests that the FabZ-ACP complex adopts the same stoichiometry in solution as observed in the crystal structure. Subsequently, a titration-based microscale thermophoresis assay (MST) was employed to identify the binding affinities of holo-ACP to FabZ (Figure 5F and 5G) [40]. On the basis of the stoichiometry, the binding curve appears in triphasic phases with binding affinities of $0.354 \pm 0.056,16.2 \pm$ 1.5 and $297 \pm 22.1 \mu \mathrm{M}$ (Figure 5F). The distinct binding affinities also indicate that the binding of ACP to FabZ hexamer involves a complicated allosteric crosstalk, not only between monomers within a FabZ dimer subunit observed from the structure, but also between dimers within the FabZ hexamer. It is likely that the binding of each ACP induces significant conformational changes of ACP-unbound and -bound FabZ dimer subunits, thus influencing the subsequent ACP binding (lower binding affinities). Such conformational changes are most likely regulated by the gatekeeper residue Tyr100, as ACP binding as well as FabZ enzymatic activity are abolished by FabZ Y100A mutagenesis (Figure 5G) [40]. Although the allosteric crosstalk between FabZ dimer subunits induced by successive ACP binding could not be observed from the current structure as the crystal was obtained under the condition of saturated ACP binding of FabZ hexamer (ACP:FabZ = 7:1 before gel-filtration purification), the solution-based biophysical results uncovered the existence of such allosteric crosstalk. Nevertheless, the current structure shows monomer-monomer allosteric crosstalk upon the asymmetric binding of ACP on FabZ dimer subunit, and reveals the dynamic seesaw-like catalytic mechanism of DH FabZ in the FAS system for the first time, which is regulated by the critical gatekeeper residue Tyr100.

\section{Discussion}

To date, there is little structural information for the 
A

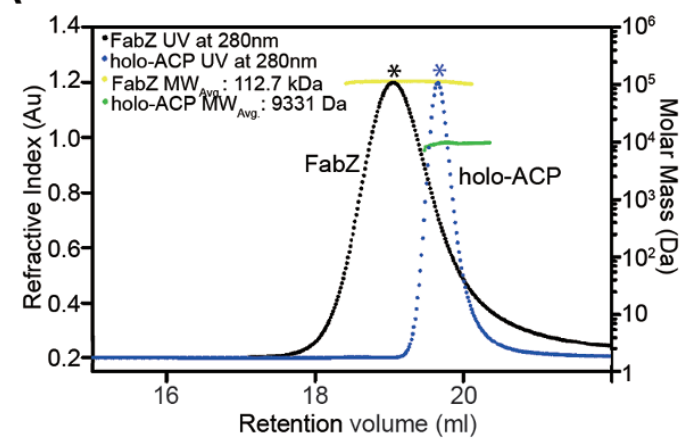

C

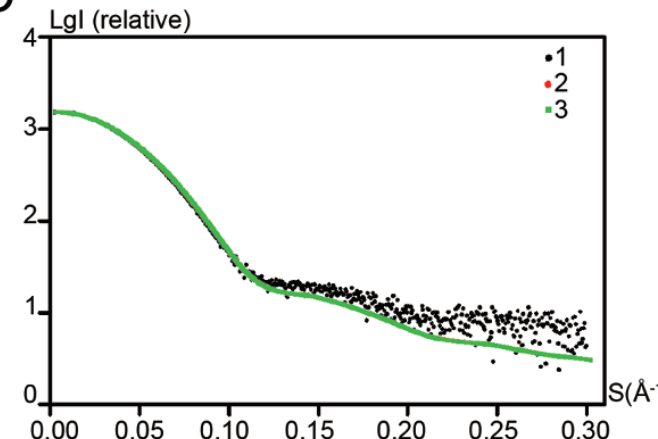

$\mathrm{B}$

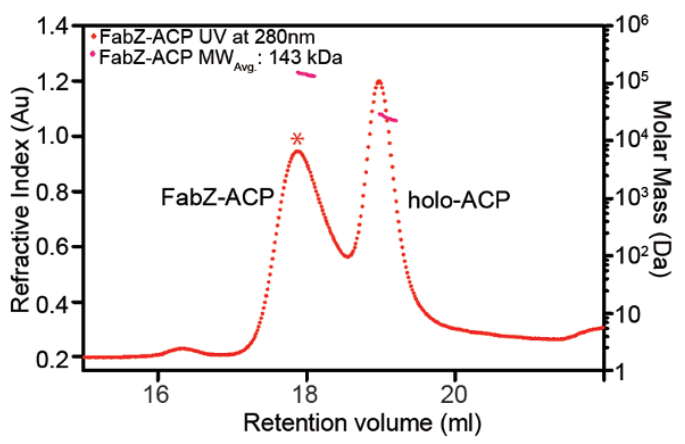

D

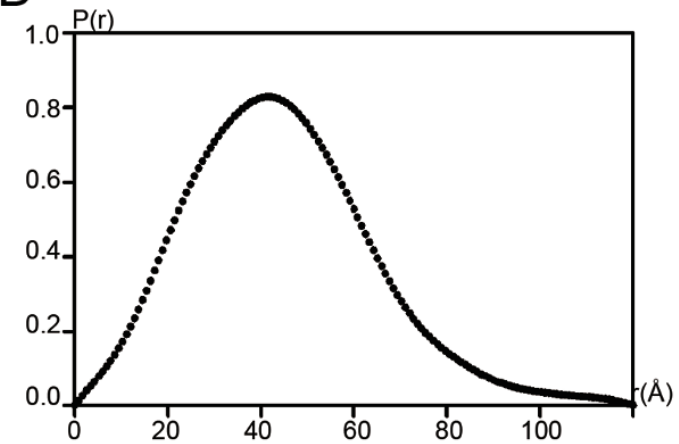

E

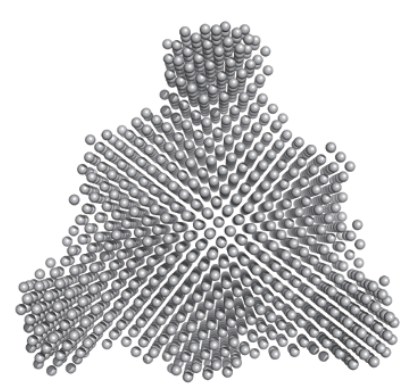

$-\left(\forall 90^{\circ}\right.$
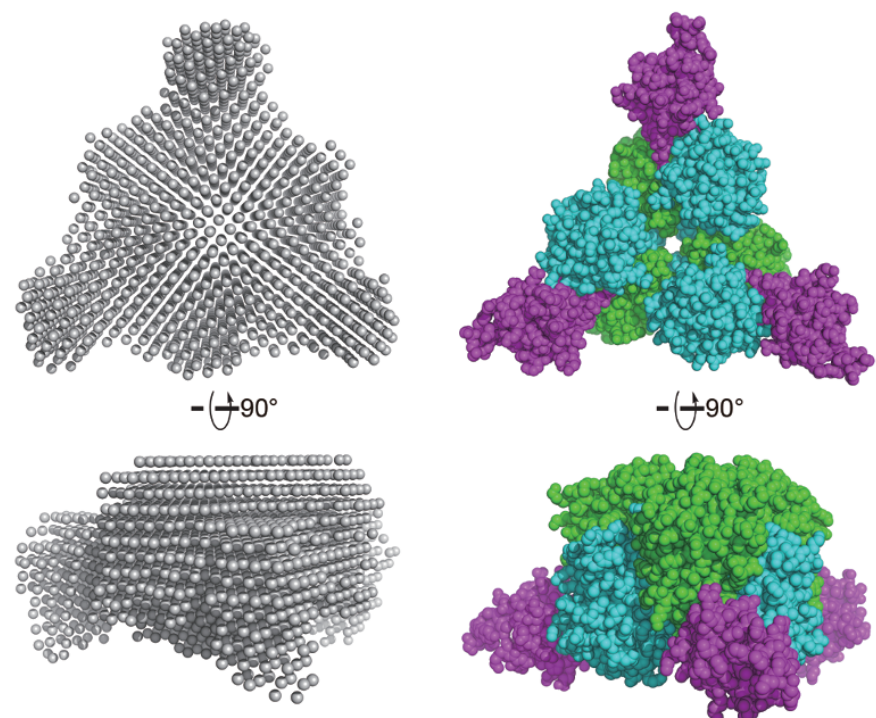

$-\left(\forall 90^{\circ}\right.$
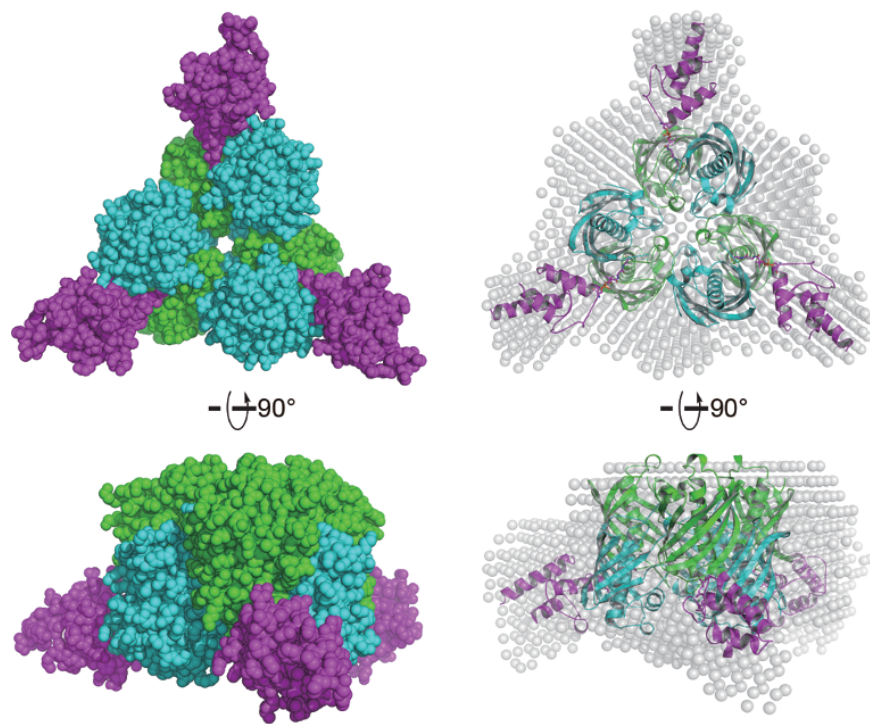

F

G
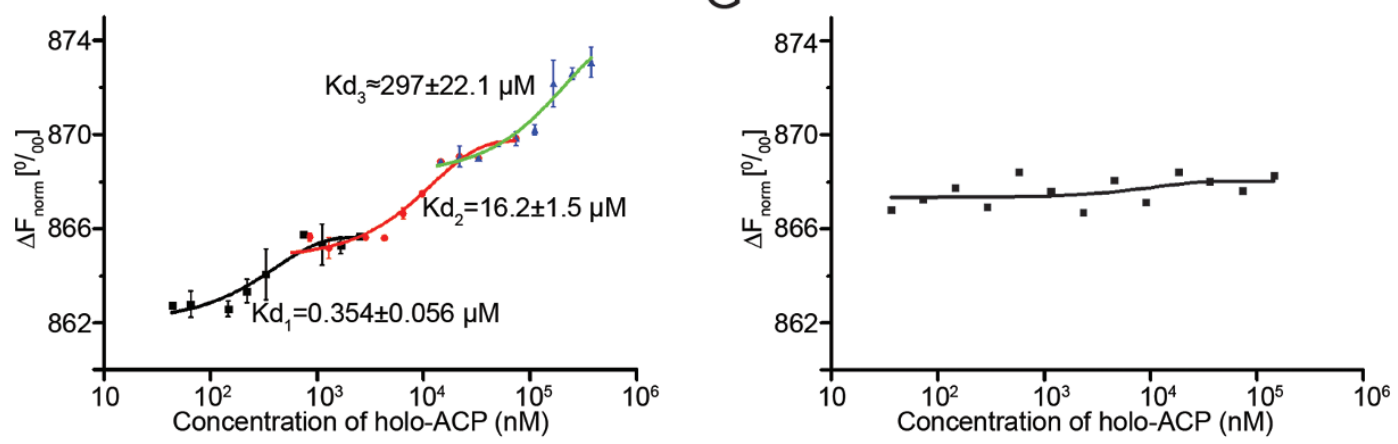

SPRINGER NATURE | Cell Research | Vol 26 No 12 | December 2016 
interaction of ACP with enzymatic modules in the FAS system, except for an incomplete FabI-ACP structure and the covalently crosslinked FabA-ACP structure [25, 29]. Our study presents the first native high-resolution enzymatic module-ACP complex structure of a FAS system. The complex exhibits a highly symmetrical FabZ hexamer- $\mathrm{ACP}_{3}$ stoichiometry with each $\mathrm{ACP}$ binding to a FabZ dimer subunit. Further structural analysis suggests that FabZ contacts ACP through $\mathrm{H}$-bonds between a constellation of conserved Arg/Lys residues near its active tunnel entrance and negatively charged residues along an ACP $\alpha 2$ helix as well as hydrophobic interactions between residues located in the hydrophobic patch near the tunnel entrance of FabZ and residues in the ACP $\alpha 2$ helix. Remarkably, the conserved Arg110 of FabZ contributes to the FabZ-ACP interactions, suggesting an important role in recognizing and binding of ACP to FabZ. The binding of ACP to FabZ also induces the movements of the $\beta$-sheet layer, which enlarges the narrow L-shaped active tunnel to a Y-shape for long fatty acid substrate accommodation. This Y-shaped tunnel has significantly more steric hindrance in reversion of trans-2-acyl-ACP to cis-3-acyl-ACP during isomerization compared with the L-shaped tunnel of FabA, which explains the absence of isomerase activity in FabZ. More importantly, the movements of the $\beta$-sheet layer not only occur on ACP-bound FabZ monomers but also on ACP-unbound monomers with even larger conformational changes, forming an unfavorable conformation for ACP binding by introducing clashes between FabZ and ACP. Additional biophysical methods, including SEC-MALS, SAXS and MST, confirmed these observations, and the corresponding binding affinities of ACP toward FabZ were determined. These findings suggest a novel dynamic seesaw-like continuous catalytic mechanism of DH FabZ with ACP in the FAS-II system, in which ACP's alternate binding is regulated by a FabZ gatekeeper residue Tyr100 through manipulating the movements of FabZ's $\beta$-sheet layer (Figure 6, and Supplementary information, Movie). Furthermore, the MST titration result suggests that the binding of ACP to FabZ involves a series of complicated allosteric crosstalk both between monomer subunits and between dimer subunits. The current crystal structure successfully shows the monomer-monomer crosstalk due to the asymmetric binding of ACP on FabZ dimer subunit. The current study mainly focuses on the structural changes within a single FabZ dimer subunit as well as the corresponding seesaw-like allosteric crosstalk mechanism on regulating ACP binding; and thus the current structural information is not adequate to explain entire allosteric changes between dimer subunits.

The superposition of FabZ-ACP structure, FabA-ACP structure (PDB code: $4 \mathrm{KEH}$ ) and the mammalian $\mathrm{DH}$ module from $S$. scrofa FASN (PDB code: 2 vz8) exhibits high overall structural similarity, indicating that these DH (module) may employ a conserved mechanism to regulate ACP catalysis (Supplementary information, Figure S6A-S6C and S10). The major difference among the structures is the numbers of ACP bound to the DH (module). In FabA-ACP structure case, two ACP mole-

Figure 5 Biophysical studies of FabZ-ACP complex in solution. (A) Size-exclusion chromatography (SEC) coupled with multi-angle light scattering (MALS) analysis. FabZ protein (black curve) or holo-ACP (blue curve) was separated using a Wyatt Technology WTC-030S5 column. The running buffer contains 20 mM HEPES (pH 8.0), $500 \mathrm{mM} \mathrm{NaCl}$ and 10 mM DTT. $500 \mathrm{mM} \mathrm{NaCl}$, instead of $150 \mathrm{mM} \mathrm{NaCl}$ was used due to the instability of FabZ protein in a low-salt buffer. Corresponding average molecular weight of the fraction peaks marked with stars was evaluated and analyzed using Wyatt Technology SECMALS system. The chromatogram shows the readings of UV detector, and $280 \mathrm{~nm}$ absorption scaling is shown in $Y$ axis on the left. The yellow and green curves indicate the calculated molecular masses of FabZ and holo-ACP throughout the peaks. The molecular mass scaling is shown in the $Y$ axis on the right. (B) SEC-MALS analysis of FabZ-ACP complex. The FabZ-ACP complex saturated with excess amounts of holo-ACP (red curve) was separated using a Wyatt Technology WTC$030 S 5$ column. The running buffer for separating the complex contains $20 \mathrm{mM}$ HEPES (pH 8.0$), 150 \mathrm{mM} \mathrm{NaCl}$ and $10 \mathrm{mM}$ DTT. The magenta curves indicate the calculated molecular masses of FabZ-ACP complex and holo-ACP throughout the peaks. (C) SAXS scattering data from FabZ-ACP complex in solution. Curve-1 (black dots): SAXS scattering experimental data. Curve-2 (red line): scattering pattern calculated from the crystal structure. Curve-3 (green line): smooth curve back transformed from the $p(r)$ and extrapolated to a zero scattering angle for the complex in solution. (D) SAXS data analysis: distance distribution functions for the complex. (E) SAXS modeling: low-resolution envelope for the complex was shown as a particle model and was superposed with the complex crystal structure. (F) Microscale thermophoresis (MST) measurements of holo-ACP binding to wild-type FabZ. Data points colored in black, red and blue indicate the differences in normalized fluorescence generated by various concentrations of holo-ACP binding to FabZ. Curves colored in black, red and green indicate the calculated fits according to the data points, and the corresponding Kd values are shown. (G) MST measurements of holo-ACP binding to FabZ (Y100A) mutant. Data points in black indicate the difference in normalized fluorescence generated by various concentrations of holo-ACP binding to FabZ (Y100A). Curves colored in black indicate the calculated fits according to the data points. 

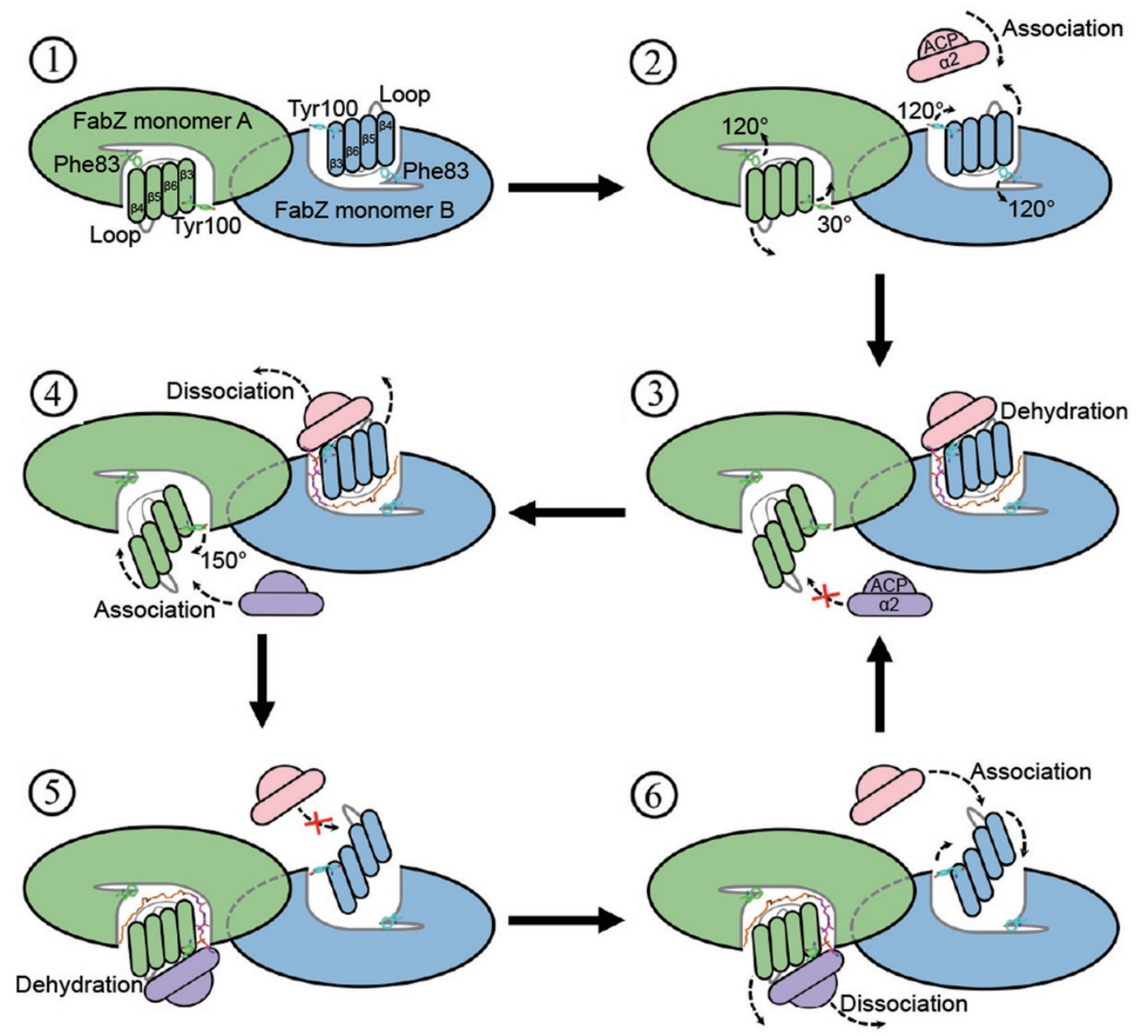

Figure 6 Schematic diagram of the dynamic seesaw-like continuous catalytic mechanism model of FabZ during fatty acid biosynthesis. Monomers A and B within a FabZ dimer subunit and two ACP molecules are colored in green, light blue, wheat and purple, respectively. Four critical $\beta$-strands from the $\beta$-sheet layer are shown as oval rafts. Key gatekeeper residues Tyr100 and Phe83 from FabZ monomer B (cyan) and monomer A (green) are shown as sticks. The 4'-phosphopantetheine arm (magenta) attached with a long fatty acid chain (orange) from ACP are shown as lines. In the native conformation of FabZ, the sidechains of Tyr100 and Phe83 adopt close conformation to protect the hydrophobic catalytic tunnel from bulk solvent (step 1). When the first ACP molecule (wheat) binds to FabZ monomer $B$, the L-shaped active tunnel is expanded to a $Y$-shape due to movement of the $\beta$-sheet layer toward 4. The sidechains of Tyr100 and Phe83 flop $\sim 120^{\circ}$ to an open conformation, which allows the substrate chain carried by the 4'-Pan-arm to insert into the active tunnel for catalytic dehydration. Meanwhile, the $\beta$-sheet layer in the ACP-unbound FabZ monomer A moves further toward $\beta 4$ due to a $\sim 30^{\circ}$ rotation of the Tyr100 sidechain from its normal closed conformation to form an abnormal conformation unfavorable for ACP binding (purple) (steps 2 and 3 ). When the dehydration reaction is finished on monomer $B$, the $\beta$-sheet layer moves further toward $\beta 4$ to release ACP, whereas the $\beta$-sheet layer on monomer $A$ moves back with $a \sim 150^{\circ}$ rotation of the Tyr100 sidechain to a normal open conformation to accept binding and catalysis of the second ACP (steps 4 and 5). By doing so, the catalysis core is exchanged from FabZ monomer $B$ to monomer $A$. When the second dehydration reaction is finished, the catalysis core is exchanged to $B$ again like a see-saw due to the movements of the $\beta$-sheet layer (steps 4-6), which causes the high-efficiency catalytic dehydration mechanism of FabZ in the FAS system.

cules bind to a FabA dimer, whereas in our structure, one ACP molecule binds to a FabZ dimer subunit. This could be caused by the covalent crosslinking of ACP to FabA. In the DH domain case, it is a pseudo-dimer with distinct differences between the two subdomains, and can only catalyze one ACP at a time rather than two. However, the DH domain shares a highly structurally conserved $\beta$-sheet layer with FabZ, where ACP molecule binds to, suggesting that the DH domain is very likely to utilize similar mechanism to regulate ACP by manipulating the 
conformational changes of the $\beta$-sheet layer. Hence, our finding also contributes to the understanding of mammalian FAS catalytic mechanism, and provides a potential drug discovery strategy against human diseases associated with FAS by inhibiting its enzymatic activity through interfering ACP-enzymatic module interactions. Collectively, our results illustrate a novel and dynamic ACP binding and regulation mechanism for the $\mathrm{DH}$ module in the FAS system, which provides a plausible explanation for previously established phenomenon and facilitates a general understanding of the biological functions of the FAS system.

Increasing evidence suggests that overproduction of fatty acids by the FAS system is correlated with MetS and various cancers [12-17, 41]. Inhibition of FAS activity significantly induces tumor cell apoptosis, suppresses tumor cell metastasis and improves insulin sensitivity [33, $42,43]$. Hence, hFASN is considered to be a promising anti-MetS and anti-tumor drug target for clinical therapy, and several specific inhibitors for interfering with the enzymatic activity of hFASN have been developed [3, 4146]. Unfortunately, most of these compounds have limited applications in clinical scenarios due to severe side effects or modest potency [23, 33]. The dynamic ACP binding and regulation mechanism of the DH module in the FAS system provides a potential novel drug design strategy for interfering with ACP-enzymatic module interactions rather than the enzymatic activities in the FAS system, which may significantly reduce side effects. Our findings may benefit clinical drug and therapy development against pathogenic infection, as well as human diseases associated with the deregulation of this pathway, including obesity, diabetes, and prostate and breast cancers.

\section{Materials and Methods}

\section{Protein subcloning, expression and purification}

Expressions and purifications of apo-ACP, holo-ACP, FabZ and FabZ (Y100A) from H. pylori were expressed and purified as described previously [36, 37]. Briefly, FabZ gene was subcloned to PQE30 plasmid and expressed in Escherichia coli strain M15 cells. QuikChange Site-Directed Mutagenesis (Stratagene) strategy was used to perform the FabZ (Y100A) mutagenesis. FabZ and FabZ (Y100A) protein were purified by using Ni-NTA and Gel-filtration column. $A C P$ gene was subcloned to pET32a vector and expressed in E. coli BL21 cells. The protein was purified by using Ni-NTA, and the N-terminal thioredoxin-His tag was removed by recombinant enterokinase protease cleavage at $4{ }^{\circ} \mathrm{C}$ for overnight. Subsequently, ACP protein was further purified by GE HiLoad 16/600 Superdex 75 prep grade gel-filtration column. The final concentration was quantified by Bradford reagent purchased from Bio-Rad. For the FabZ-holo-ACP complex, the purified FabZ and holo-ACP protein were mixed in molar ratio 1:7, and dialyzed against $20 \mathrm{mM}$ HEPES, pH 8.0, $150 \mathrm{mM} \mathrm{NaCl}$ and $10 \mathrm{mM}$ DTT. The mixture was concentrated and loaded to HiLoad 16/600 Superdex 200 prep grade column for further purification. The complex peak was pooled and concentrated to $10 \mathrm{mg} / \mathrm{ml}$.

Apo-ACP, holo-ACP and FabZ-ACP complex crystallization Approximately $10 \mathrm{mg} / \mathrm{ml}$ apo-ACP, holo-ACP and complex protein were mixed with an equal volume of reservoir solution containing 1.0-3.0 M sodium chloride and 0.1 M sodium acetate, and 1.0-3.0 $\mathrm{M}$ lithium sulfate and $0.5 \mathrm{M}$ ammonium sulfate, and 10\%-30\% PEG3350, $0.05 \mathrm{M}$ tri-sodium citrate dehydrate, respectively, and equilibrated against $500 \mu \mathrm{l}$ of the reservoir solution at $277 \mathrm{~K}$. Crystals appeared within 30 days. The crystals were then flash-frozen in liquid nitrogen with $20 \%-30 \%$ glycerol (v/v) as the cryoprotectant solution.

\section{Data collection and structure determination}

Crystal diffraction data of apo-ACP and FabZ-ACP complex were collected at beamline BL19U1 at the National Center for Protein Sciences Shanghai and Shanghai Synchrotron Radiation Facility, China. Significant decay of the complex crystals during data collection caused the resolution cut off at $2.55 \AA$. While the crystal diffraction data of holo-ACP were collected at $100 \mathrm{~K}$ using $\mathrm{CuK} \alpha \mathrm{X}$-ray with a Rigaku R-AXIS IV++ image plate. The data were further processed using HKL2000 software package [47] and CrystalClear software. The structures were solved by MR with the published structure of apo-EcACP (PDB code: 1T8K) and HpFabZ (PDB code: 2GLL) as search models, and refined by using programs Phenix [48] (complex) and CNS (apo and holo-ACP). The crystallographic statistics are summarized in Supplementary information, Table S1. Electron density interpretation and model building were performed using the computer graphics program Coot [49]. Final structures were visualized by PyMol software.

\section{Covalent docking}

SYBYL package (www.tripos.com) was used to prepare the fatty acid chains and the protein structures used in docking. Initial structures of fatty acid chains in different lengths (4- to 16-carbon chain) were sketched using the SYBYL program with standard geometric parameters, and Gasteiger-Hucker charge was added. All fatty acids were then optimized using the standard TRIPOS force field with Gasteiger-Hückel charges until the energy gradient converged to below $0.05 \mathrm{kcal} / \mathrm{mol}$. Waters in the crystal structure of FabZ were removed, and all hydrogens were added. Kollman all-atom charges were assigned for FabZ and Gasteiger-Hückel charge was computed for residue 36 with 4'-Pan-arm.

Genetic algorithm-based docking program GOLD was used for molecular docking simulations [50]. Fatty acid chain-binding site for docking was defined as a collection of all atoms within 25 $\AA$ around the midpoint of the terminal S of the 4-Pan-arm and $\mathrm{O}$ atom of Lys97, so the active site would be big enough to include the possible binding sites of variable length fatty acid chains. The GOLDScore fitness function was employed in scoring conformations. The parameters for GOLD were as follows: numbers of GA ran 50, no early termination was allowed and all other parameters were used as default (Supplementary information, Table S3).

Size-exclusion chromatography coupled with multi-angle light scattering measurements 
Approximately $10 \mu 1,0.5 \mathrm{mg} / \mathrm{ml}$ purified FabZ-ACP complex saturated with excess amount of holo-ACP sample was injected into HPLC system (Agilent Technologies) and separated by using Wyatt Technology WTC-030S5 column with running buffer containing $20 \mathrm{mM}$ HEPES, $\mathrm{pH} 8.0,150 \mathrm{mM} \mathrm{NaCl}$ and $10 \mathrm{mM}$ DTT. The native FabZ or holo-ACP protein was separated with running buffer containing $20 \mathrm{mM}$ HEPES, $\mathrm{pH} 8.0,500 \mathrm{mM} \mathrm{NaCl}$ and 10 mM DTT due to the instability of FabZ protein under low salt (150 $\mathrm{mM} \mathrm{NaCl}$ ) buffer. Corresponding molecular weight of the fraction peaks was evaluated and analyzed by using Wyatt Technology SEC-MALS system, which was calibrated by using $2 \mathrm{mg} / \mathrm{ml}$ standard BSA sample. As shown in Figure 5A and 5B, FabZ-ACP $\mathrm{MW}_{\text {calculated }}=145 \mathrm{KDa}, \mathrm{MW}_{\text {observed }}=143( \pm 2.0 \%) \mathrm{kDa} ; \mathrm{FabZ}$ $\mathrm{MW}_{\text {calculated }}=117 \mathrm{kDa}, \mathrm{MW}_{\text {observed }}=112.7( \pm 0.66 \%) \mathrm{kDa}$; holo-ACP $\mathrm{MW}_{\text {calculated }}=9382 \mathrm{Da}, \mathrm{MW}_{\text {observed }}=9331( \pm 4.3 \%) \mathrm{Da}$.

\section{Synchrotron solution small angle X-ray scattering measure-} ments

FabZ-ACP complex purified from gel-filtration in the solution containing $20 \mathrm{mM}$ HEPES, pH 8.0, $150 \mathrm{mM} \mathrm{NaCl}$ and $10 \mathrm{mM}$ DTT was used in the Synchrotron SAXS experiment. The measurement was performed in a vacuum cuvette with exposure time of $1 \mathrm{~s}$ to diminish the parasitic scattering under wavelength $1.03 \AA$ at National Center for Protein Sciences Shanghai (NCPSS) beamline BL19U2, China. The scattering was recorded in the range of the momentum transfer $0.015<\mathrm{s}<0.420 / \AA$, where $\mathrm{s}=(4 \pi \times$ $\sin \theta) / \lambda$ and $2 \theta$ is the scattering angle. SAXS data were subsequently processed and analyzed by using the ATSAS program suite [51]. Briefly, program PRIMUS [52] was employed to process the experimental scattering data and correct the solvent background scattering. Program CRYSOL was used to generate the theoretical scattering from the FabZ-ACP complex crystal structure [53]. Program GNOM was employed to calculate the distance distribution function $\mathrm{p}(\mathrm{r}$ ) (Figure 5C and 5D) [54]. Subsequently, programs DAMMIN [55], GASBOR [56] and DAMAVER [57] were used to reconstruct the low-resolution shapes to a dummy atom particle model, and superpose to the crystal structure (Figure 5E). The figure was generated by using GraphPad Prism 5 and PyMol software.

\section{Microscale thermophoresis assays}

MST assay is a new immobilization-free and titration-based technology for biomolecule interaction determination [58]. The assay was performed with a NanoTemper Monolith NT.115 instrument (NanoTemper Technologies GmbH). Briefly, FabZ or FabZ (Y100A) was labeled with fluorescence dye NT-647 (amine-reaction) according to manufacturer's protocol. Subsequently, 50 $\mathrm{nM}$ of labeled protein was mixed with various concentrations of holo-ACP in the reaction buffer containing $20 \mathrm{mM}$ HEPES, $\mathrm{pH} 8.0$, $150 \mathrm{mM} \mathrm{NaCl}, 5 \mathrm{mM}$ fresh DTT and $0.05 \%$ Tween 20 . After incubation for $5 \mathrm{~min}$ under room temperature, $10 \mu \mathrm{l}$ of the mixtures were loaded into premium coated capillaries for measurement. The MST data were then collected under $40 \%$ infrared laser power and $20 \%$ light-emitting diode power. Nanotemper analysis software (1.5.41) was used to fit the independent experimental data and determine $\mathrm{Kd}$ and standard deviation value. The figure was generated by using GraphPad Prism 5.

\section{Acknowledgments}

This project was supported by the National Natural Science Foundation of China (21572133 to LZ, 21210003 to HJ and 91413102 to XS), the Program for Professors of Special Appointment (Eastern Scholar) at the Shanghai Institutions of Higher Learning (to LZ), the National Great Basic Science Project of China (2010CB529806 to HC), the Ministry of Science and Technology of China (2015CB910304 to HJ). We thank the staff of beamlines BL19U1 and BL19U2 at the National Center for Protein Sciences Shanghai (NCPSS) and Shanghai Synchrotron Radiation Facility (SSRF, China) for assistance with crystal diffraction and SAXS data collection. We thank professors Jianping Ding and Jiamu Du from Shanghai Institutes for Biological Sciences, Chinese Academy of Sciences for data analysis and discussion. We thank Mr Jing Lan from WYATT Technology for SEC-MALS technical support, Mr Fenfei Wang from Quantum Design China \& NanoTemper Technologies GmbH for MST technical support, and Dr José Ramón Andrade Candelario for production of the animation (http://www.3dciencia.com/).

\section{Author Contributions}

HJ and LZ designed the experiments; LZ, TRF and ZWC performed protein purification; LZ and LZ performed the crystallization as well as SEC-MALS, SAXS and the MST biophysical experiments; JFX and JRX performed the computational analysis; HJ, LZ, LZ and JFX wrote the paper. All authors discussed and commented on the manuscript.

\section{Competing Financial Interests}

The authors declare no competing financial interests.

\section{References}

1 Smith S, Witkowski A, Joshi AK. Structural and functional organization of the animal fatty acid synthase. Prog Lipid Res 2003; 42:289-317.

2 White SW, Zheng J, Zhang YM, Rock. The structural biology of type II fatty acid biosynthesis. Annu Rev Biochem 2005; 74:791-831.

3 Cronan JE, Thomas J. Bacterial fatty acid synthesis and its relationships with polyketide synthetic pathways. Methods Enzymol 2009; 459:395-433.

4 Anghel SI, Wahli W. Fat poetry: a kingdom for PPAR gamma. Cell Res 2007; 17:486-511.

5 Clay HB, Parl AK, Mitchell SL, Singh L, Bell LN, Murdock DG. Altering the mitochondrial fatty acid synthesis (mtFASII) pathway modulates cellular metabolic states and bioactive lipid profiles as revealed by metabolomic profiling. PLoS One 2016; 11:e0151171.

6 Wu J, Sun Y, Zhao Y, et al. Deficient plastidic fatty acid synthesis triggers cell death by modulating mitochondrial reactive oxygen species. Cell Res 2015; 25:621-633.

7 Ronnett GV, Kim EK, Landree LE, Tu Y. Fatty acid metabolism as a target for obesity treatment. Physiol Behav 2005; 85:25-35.

8 Cooke AA, Connaughton RM, Lyons CL, McMorrow AM, Roche HM. Fatty acids and chronic low grade inflammation associated with obesity and the metabolic syndrome. Eur $J$ Pharmacol 2016; 785:207-214. 
9 Taylor EM, Jones AD, Henagan TM. A review of mitochondrial-derived fatty acids in epigenetic regulation of obesity and type 2 diabetes. J Nutrit Health Food Sci 2014; 2:1-4.

10 Zhang Y, Kent JW, Lee A, et al. Fatty acid binding protein 3 (fabp3) is associated with insulin, lipids and cardiovascular phenotypes of the metabolic syndrome through epigenetic modifications in a Northern European family population. BMC Med Genomics 2013, 6:9-22.

11 Arumugam S, Sreedhar R, Thandavarayan RA, Karuppagounder V, Watanabe K. Targeting fatty acid metabolism in heart failure: is it a suitable therapeutic approach. Drug Discov Today 2016; 21:1003-1008.

12 Kuhajda FP. Fatty acid synthase and cancer: new application of an old pathway. Cancer Res 2006; 66:5977-5980.

13 Menendez JA, Lupu R. Fatty acid synthase and the lipogenic phenotype in cancer pathogenesis. Nat Rev Cancer 2007; 7:763-777.

14 Rossi S, Graner E, Febbo P, et al. Fatty acid synthase expression defines distinct molecular signatures in prostate cancer. Mol Cancer Res 2003; 1:707-715.

15 Migita T, Ruiz S, Fornari A, et al. Fatty acid synthase: a metabolic enzyme and candidate oncogene in prostate cancer. $J$ Natl Cancer Inst 2009; 101:519-532.

16 Vazquez-Martin A, Colomer R, Brunet J, Lupu R, Menendez JA. Overexpression of fatty acid synthase gene activates HER1/HER2 tyrosine kinase receptors in human breast epithelial cells. Cell Prolif 2008; 41:59-85.

17 Jin Q, Yuan LX, Boulbes D, et al. Fatty acid synthase phosphorylation: a novel therapeutic target in HER2-overexpressing breast cancer cells. Breast Cancer Res 2010; 12:R96-R110.

18 Jenke-Kodama H, Sandmann A, Müller R, Dittmann E. Evolutionary implications of bacterial polyketide synthases. Mol Biol Evol 2005; 22:2027-2039.

19 Maier T, Jenni S, Ban N. Architecture of mammalian fatty acid synthase at 4.5 A resolution. Science 2006; 311:12581262.

20 Jenni S, Leibundgut M, Maier T, Ban N. Architecture of a fungal fatty acid synthase at 5 A resolution. Science 2006; 311:1263-1267.

21 Lomakin IB, Xiong Y, Steitz TA. The crystal structure of yeast fatty acid synthase, a cellular machine with eight active sites working together. Cell 2007; 129:319-332.

22 Jenni S, Leibundgut M, Boehringer D, Frick C, Mikolásek B, Ban N. Structure of fungal fatty acid synthase and implications for iterative substrate shuttling. Science 2007; 316:254261.

23 Maier T, Leibundgut M, Ban N. The crystal structure of a mammalian fatty acid synthase. Science 2008; 321:13151322.

24 Cronan JE, Thomas J. Bacterial fatty acid synthesis and its relationships with polyketide synthetic pathways. Methods Enzymol 2009; 459:395-433.

25 Rafi S, Novichenok P, Kolappan S, et al. Structure of acyl carrier protein bound to FabI, the FASII enoyl reductase from Escherichia coli. J Biol Chem 2006; 281:39285-39293.

26 Zhang L, Liu W, Hu T, et al. Structural basis for catalytic and inhibitory mechanisms of beta-hydroxyacyl-acyl carrier protein dehydratase (FabZ). J Biol Chem 2008; 283:5370-5379.
27 Kimber MS, Martin F, Lu Y, et al. The structure of (3R)-hydroxyacyl-acyl carrier protein dehydratase (FabZ) from $\mathrm{Pseu}$ domonas aeruginosa. J Biol Chem 2004; 279:52593-52602.

28 Kostrewa D, Winkler FK, Folkers G, Scapozza L, Perozzo R. The crystal structure of PfFabZ, the unique beta-hydroxyacyl-ACP dehydratase involved in fatty acid biosynthesis of Plasmodium falciparum. Protein Sci 2005; 14:1570-1580.

29 Nguyen C, Haushalter RW, Lee DJ, et al. Trapping the dynamic acyl carrier protein in fatty acid biosynthesis. Nature 2014; 505:427-431.

30 Zeng D, Zhao J, Chung HS, Guan Z, Raetz CR, Zhou P. Mutants resistant to LpxC inhibitors by rebalancing cellular homeostasis. J Biol Chem 2013; 288:5475-5486.

31 Heath RJ, White SW, Rock CO. Lipid biosynthesis as a target for antibacterial agents. Prog Lipid Res 2001; 40:467-497.

32 Campbell JW, Cronan JE Jr. Bacterial fatty acid biosynthesis: targets for antibacterial drug discovery. Annu Rev Microbiol 2001; 55:305-332.

33 Orita H, Coulter J, Lemmon C, et al. Selective inhibition of fatty acid synthase for lung cancer treatment. Clin Cancer Res 2007; 13:7139-7145.

34 He L, Zhang L, Liu X, et al. Discovering potent inhibitors against the beta-hydroxyacyl-acyl carrier protein dehydratase (FabZ) of Helicobacter pylori: structure-based design, synthesis, bioassay, and crystal structure determination. J Med Chem 2009; 52:2465-2481.

35 Hardwicke MA, Rendina AR, Williams SP, et al. A human fatty acid synthase inhibitor binds $\beta$-ketoacyl reductase in the keto-substrate site. Nat Chem Biol 2014; 10:774-779.

36 Liu W, Luo C, Han C, et al. A new beta-hydroxyacyl-acyl carrier protein dehydratase (FabZ) from Helicobacter pylori: molecular cloning, enzymatic characterization, and structural modeling. Biochem Biophys Res Commun 2005; 333:10781086.

37 Liu W, Du L, Zhang L, Chen J, Shen X, Jiang H. Helicobacter pylori acyl carrier protein: expression, purification, and its interaction with beta-hydroxyacyl-ACP dehydratase. Protein Expr Purif 2007; 52:74-81.

38 Pasta S, Witkowski A, Joshi AK, Smith S. Catalytic residues are shared between two pseudosubunits of the dehydratase domain of the animal fatty acid synthase. Chem Biol 2007; 14:1377-1385.

39 Jones G, Willett P, Glen RC, Leach AR, Taylor R. Development and validation of a genetic algorithm for flexible docking. J Mol Biol 1997; 267:727-748.

40 Willemsen MJ, André T, Wanner R, et al. Microscale thermophoresis: interaction analysis and beyond. J Mol Struct 2014; 1077:101-113.

41 Maximino P, Horta PM, Santos LC, Oliveira CL, Fisberg M. Fatty acid intake and metabolic syndrome among overweight and obese women. Rev Bras Epidemiol 2015; 18:930-942.

42 Wen S, Niu Y, Lee SO, et al. Targeting fatty acid synthase with ASC-J9 suppresses proliferation and invasion of prostate cancer cells. Mol Carcinog 2016 Feb 19. doi: 10.1002/ mc. 22468

43 Harriman G, Greenwood J, Bhat S, et al. Acetyl-CoA carboxylase inhibition by ND-630 reduces hepatic steatosis, improves insulin sensitivity, and modulates dyslipidemia in rats. Proc Natl Acad Sci USA 2016; 113:E1796-1805. 
44 Zhang JS, Lei JP, Wei GQ, Chen H, Ma CY, Jiang HZ. Natural fatty acid synthase inhibitors as potent therapeutic agents for cancers: a review. Pharm Biol 2016; 54:1919-1925.

45 Kuhajda FP, Pizer ES, Li JN, Mani NS, Frehywot GL, Townsend CA. Synthesis and antitumor activity of an inhibitor of fatty acid synthase. Proc Natl Acad Sci USA 2000; 97:3450-3454.

46 Hardwicke MA, Rendina AR, Williams SP, et al. A human fatty acid synthase inhibitor binds $\beta$-ketoacyl reductase in the keto-substrate site. Nat Chem Biol 2014; 10:774-779.

47 Otwinowski Z, Minor W. Processing of X-ray diffraction data collected in oscillation mode. Methods Enzymol 1997; 276:307-326.

48 Adams PD, Afonine PV, Bunkóczi G, et al. PHENIX: a comprehensive Python-based system for macromolecular structure solution. Acta Cryst D 2010; 66:213-221.

49 Emsley P, Cowtan K. Coot: model-building tools for molecular graphics. Acta Cryst D 2004; 60:2126-2132.

50 Jones G, Willett P, Glen RC, Leach AR, Taylor R. Development and validation of a genetic algorithm for flexible docking. J Mol Biol 1997; 267:727-748.

51 Konarev PV, Petoukhov MV, Volkov VV, Svergun DI. ATSAS 2.1, a program package for small-angle scattering data analysis. J Appl Crystallogr 2006; 9:277-286.

52 Konarev PV, Volkov VV, Sokolova AV, Koch MHJ, Svergun DI. PRIMUS: a Windows PC-based system for small-angle scattering data analysis. J Appl Crystallogr 2003; 36:12771282.

53 Svergun DI, Barberato C, Koch MHJ. CRYSOL - a program to evaluate X-ray solution scattering of biological macromolecules from atomic coordinates. J Appl Crystallogr 1995; 28:768-773.
54 Svergun DI. Determination of the regularization parameter in indirect-transform methods using perceptual criteria. $J$ Appl Crystallogr 1992; 25:495-503.

55 Svergun DI. Restoring low resolution structure of biological macromolecules from solution scattering using simulated annealing. Biophys J 1999; 76:2879-2886.

56 Svergun DI, Petoukhov MV, Koch MHJ. Determination of domain structure of proteins from X-ray solution scattering. Biophys J 2001; 80:2946-2953.

57 Volkov VV, Svergun DI. Uniqueness of ab initio shape determination in small-angle scattering. J Appl Crystallogr 2003; 36:860-864.

58 Jerabek-Willemsen M, Wienken CJ, Braun D, Baaske P, Duhr $\mathrm{S}$. Molecular interaction studies using microscale thermophoresis. Assay Drug Dev Technol 2011; 9:342-353.

(Supplementary information is linked to the online version of the paper on the Cell Research website.)

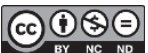

This work is licensed under a Creative Commons Attribution-NonCommercial-NoDerivs 4.0 Unported License. The images or other third party material in this article are included in the article's Creative Commons license, unless indicated otherwise in the credit line; if the material is not included under the Creative Commons license, users will need to obtain permission from the license holder to reproduce the material. To view a copy of this license, visit http://creativecommons.org/licenses/by-nc-nd/4.0/

(C) The Author(s) 2016 